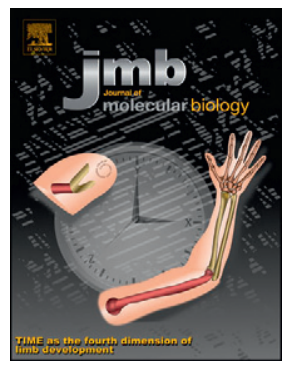

\title{
Limb Patterning: From Signaling Gradients to Molecular Oscillations
}

\author{
Caroline J. Sheeba ${ }^{1,2,3,4}$, Raquel P. Andrade ${ }^{1,2, \dagger}$ and Isabel Palmeirim ${ }^{3,4, \dagger}$ \\ 1 - Life and Health Sciences Research Institute (ICVS), School of Health Sciences, University of Minho, 4710-057 Braga, Portugal \\ 2 - ICVS/3B's-PT Government Associate Laboratory, Braga/Guimarães, Portugal \\ 3 - Regenerative Medicine Program, Departamento de Ciências Biomédicas e Medicina, Universidade do Algarve, \\ 8005-139 Faro, Portugal \\ 4 - IBB-Institute for Biotechnology and Bioengineering, Centro de Biomedicina Molecular e Estrutural, Universidade do Algarve, \\ 8005-139 Faro, Portugal
}

Correspondence to Isabel Palmeirim: Regenerative Medicine Program, Departamento de Ciências Biomédicas e Medicina,

Universidade do Algarve, 8005-139 Faro, Portugal. imesteves@ualg.pt http://dx.doi.org/10.1016/j.jmb.2013.11.022

Edited by M. Yaniv

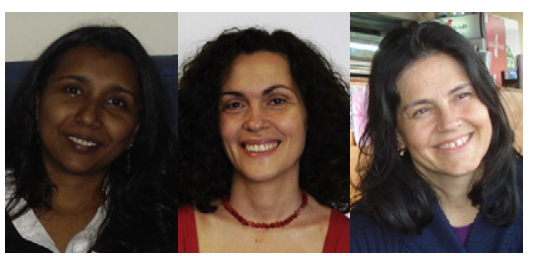

Caroline J. Sheeba, Raquel P. Andrade and Isabel Palmeirim

\begin{abstract}
The developing forelimb is patterned along the proximal-distal and anterior-posterior axes by opposing gradients of retinoic acid and fibroblast growth factors and by graded sonic hedgehog signaling, respectively. However, how coordinated patterning along both axes is accomplished with temporal precision remains unknown. The limb molecular oscillator hairy2 was recently shown to be a direct readout of the combined signaling activities of retinoic acid, fibroblast growth factor and sonic hedgehog in the limb mesenchyme. Herein, an integrated time-space model is presented to conciliate the progress zone and two-signal models for limb patterning. We propose that the limb clock may allow temporal information to be decoded into positional information when the distance between opposing signaling gradients is no longer sufficient to provide distinct cell fate specification.
\end{abstract}

(c) 2013 The Authors. Published by Elsevier Ltd. All rights reserved.

Embryo limb development has been thoroughly scrutinized in search for the molecular mechanisms involved in translating gradients of morphogen activity into positional information over time and

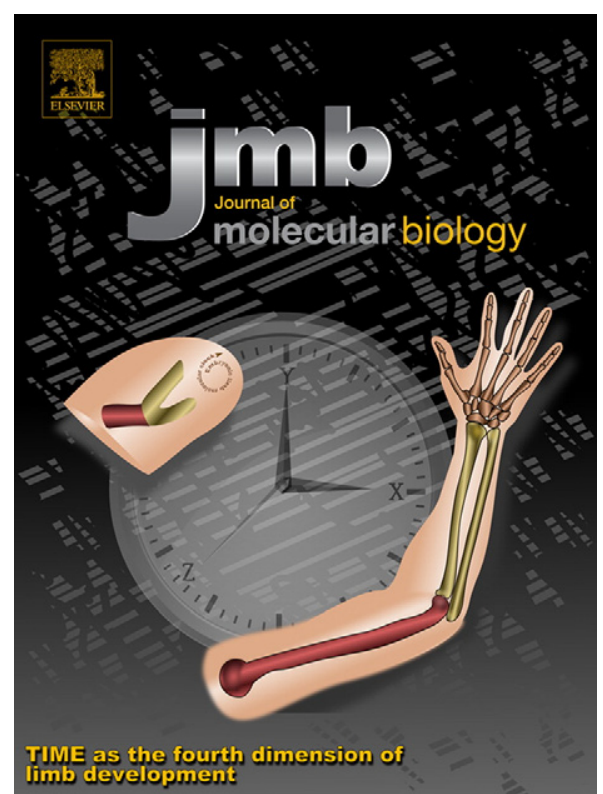

Legend. The embryo molecular clock (EC), based on hairy2 gene expression, operating in the distal limb mesenchyme has been recently identified to be influenced by the RA, FGF and SHH signaling pathways, which govern the proximaldistal and anterior-posterior limb patterning. Since the EC gene, hairy2 expression lies at the intersection of the key limb signaling pathways, we propose that the notion of time provided by EC oscillations to the distal limb progenitor cells as the fourth dimension of limb development. 
space. The limb distal apical ectodermal ridge (AER) produces fibroblast growth factors (FGFs) that drive proximal-distal (PD) outgrowth and patterning [1] via Erk/MAPK and Akt/PI3K pathway activation [2,3]. FGF activity is counteracted in the proximal limb by retinoic acid (RA) produced by the embryo flank [4]. The zone of polarizing activity (ZPA) in the posterior distal limb is a signaling center producing sonic hedgehog $(\mathrm{SHH})$, which mediates anterior-posterior (AP) limb patterning through modulation of Gli1-Gli3 transcription factor activities [5,6]. Different models for limb patterning have been proposed; however, none accounts for coordinated spatiotemporal patterning along both PD and AP axes. Two recent publications studied the dependence of hairy2 gene expression on the major signaling morphogens operating during limb development-FGF, SHH and $R A[7,8]$. The results obtained position the hairy2 molecular oscillator at the intersection of the multiple signaling activities driving limb PD and AP outgrowth and patterning.

Limb PD patterning is currently explained by the two-signal model [9]. Here, the three limb elements (stylopod, zeugopod and autopod) are established by opposing signaling gradients of AER-derived FGFs and RA produced in the embryo flank. Over time, these morphogen sources are gradually distanced in space, generating appropriate thresholds of signaling activity where the limb element boundaries are established. Data accumulated over the past years clearly show the imperative requirement of AER-FGF $[10,11]$ and flank-RA $[12,13]$ signaling, as well as their opposing activities $[14,15]$ for proper limb PD outgrowth and patterning. However, how diffuse morphogen gradients can generate limb element boundaries with such precision remains to be clarified. Importantly, time-dependent responses to these two opposing limb signaling activities have been reported $[1,15]$; distal limb cells lose their ability to respond to RA over time, even when a local source of RA is provided [15] and limb truncations resulting from AER ablation are less severe when the manipulation is performed later in time [1]. This could be explained as a consequence of irreversible cell fate commitment progressively acquired by the distal limb tissue over time. Time also assumes an important dimension in acquisition of cell identity along the limb AP axis since the duration of tissue exposure to $\mathrm{SHH}$ signaling is also a crucial factor in digit specification [16-18].

The existence of a time-counting mechanism operating in the distal limb mesenchymal cells was long proposed by the progress zone model for limb PD patterning [1]. A molecular clock in the developing limb was reported in 2007 by showing that the HES (hairy enhancer of split) hairy2 transcription factor presented gene expression oscillations in the chick distal limb with a 6-h periodicity [19], corresponding to half the time required to specify a limb skeletal element $[19,20]$. hairy2 expression appears in the presumptive forelimb region as early as stage $\mathrm{HH} 14$, is uniformly expressed in the entire limb mesenchyme until stage $\mathrm{HH} 17$ and displays positive and negative expression domains at stages $\mathrm{HH} 18 / \mathrm{HH} 19$. From $\mathrm{HH} 20$ to $\mathrm{HH}_{2} 8$, hairy2 expression oscillates in the chondrogenic precursor cells along PD and AP axes [19]. A similar molecular clock has long been known to underlie timely axial body segmentation during somitogenesis [21], and an array of other biological systems also possess functional cycles of HES gene expression [22]. During somitogenesis, synchronous HES gene oscillations function cooperatively with counteracting gradients of FGF and RA activity to translate temporal information into spatial patterns along the AP body axis [23,24]. Resende et al. further described a crucial role for notochord-derived $\mathrm{SHH}$ signaling in control of somitogenesis clock periodicity [25]. Hence, this well-established molecular clock relies on FGF, $\mathrm{RA}$ and $\mathrm{SHH}$ morphogens for its functional role in embryo body segmentation. Interestingly, these are precisely the signaling molecules overseeing limb PD and AP outgrowth and patterning, raising the hypothesis that a limb molecular clock could also be playing a role in translating temporal into spatial information in this system.

Addressing this issue, two recent publications have characterized the influence of AER-FGF, ZPA-SHH and flank-RA signaling activities on hairy2 expression in the chick forelimb bud $[7,8]$. hairy2 expression in the undifferentiated distal limb tissue closely reflects the dynamics of the limb signaling centers activities [7]. AER-FGFs were found to be short-term, short-range instructive signals on distal limb hairy2 expression through p-Erk, while ZPA-SHH acts as a long-term, long-range permissive signal [7]. Gli3, a crucial effector of SHH signaling in the developing limb $[5,26]$, oversees tissue permissiveness for hairy2 expression in such a way that FGF instructive signaling can only induce hairy2 expression in the presence of low relative amounts of Gli3 repressor form (Gli3-A/Gli3-R > 1). Consequently, hairy2 expression pattern in the distal limb mirrors Gli activation status along the AP axis of the tissue: (1) hairy2 expression is excluded from the anterior-most limb, where Gli-R activity is predominant (Gli-A/Gli-R $<1$ ). Accordingly, expression of the hairy2 homolog hes 1 gene expands anteriorly in Gli3 conditional knockout mice limbs [27], suggesting that the regulatory role of Gli3 on HES expression is a conserved trait across species; (2) ZPA-SHH ensures permanent hairy2 expression in the posterior distal limb by restricting Gli-R levels (Gli-A/Gli-R > 1); (3) in the medial region of the distal AP axis, an intermediate state of Gli activity is established and hairy2 expression is now oscillatory in nature. Proximal RA was further shown to positively regulate hairy2 expression as both instructive and permissive signals by activating p-Erk and relieving Gli-R-mediated inhibition, respectively, while BMP4 inhibited hairy2 [8]. Combining the effects 
observed for FGF, RA and SHH on hairy2 expression, the emerging overall picture is that the spatial distribution of these morphogen gradients and resulting intracellular signaling activities matches the expression patterns/domains of hairy2 over time in limb development (Fig. 1). Recent studies have elegantly shown that morphogen gradients are capable of generating distinct patterns of transcription factor gene expression, ranging from stripes to oscillations, and suggest that this mechanism could underlie the somitogenesis molecular clock $[28,29]$. Considering that hairy2 off/ oscillatory/on states result from graded morphogen activities, it is tempting to postulate that a similar mechanism could also generate hairy2 oscillations in the limb. The molecular components of a putative gene regulatory network operating in the limb remain to be identified, and our data suggest that Hairy2 could be a candidate. However, further knowledge of Hairy2 action on the limb signaling molecules such as $\mathrm{SHH}$, Gremlin, BMPs and FGFs will be crucial for establish- ing Hairy2 as a core component of such network. Variations in Erk phosphorylation levels in the distal limb could also underlie oscillations of hairy2 expression, as they are known to drive hes gene oscillations in both mouse PSM [30] and C3H10T1/2 mesenchymal stem cells [31].

We propose that, in the early limb bud (Fig. 1a), RA (permissive and instructive) and FGF8 (instructive) signaling underlie constant hairy2 expression. BMP signaling is suppressed by RA $[32,33]$ and by Gremlin (GREM) [34], ensuring low levels of Gli-R and constant hairy2 expression. Over time, the distal limb becomes progressively distanced from the RA source and is protected from RA influence by FGF induced cyp26 expression [35]. Under these conditions (Fig. 1b), BMP4 in the anterior distal limb is relieved from RA-mediated inhibition [32,33], favoring Gli3-R activity [36] and Gli3-A/Gli3-R $<1$, which inhibits hairy2 expression. Gli3-R induces bmp4 [37] and excludes grem expression in the anterior limb [38-40], further

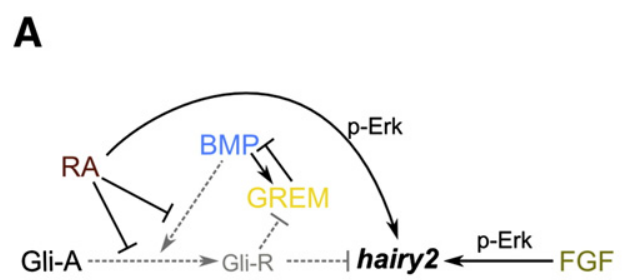

B
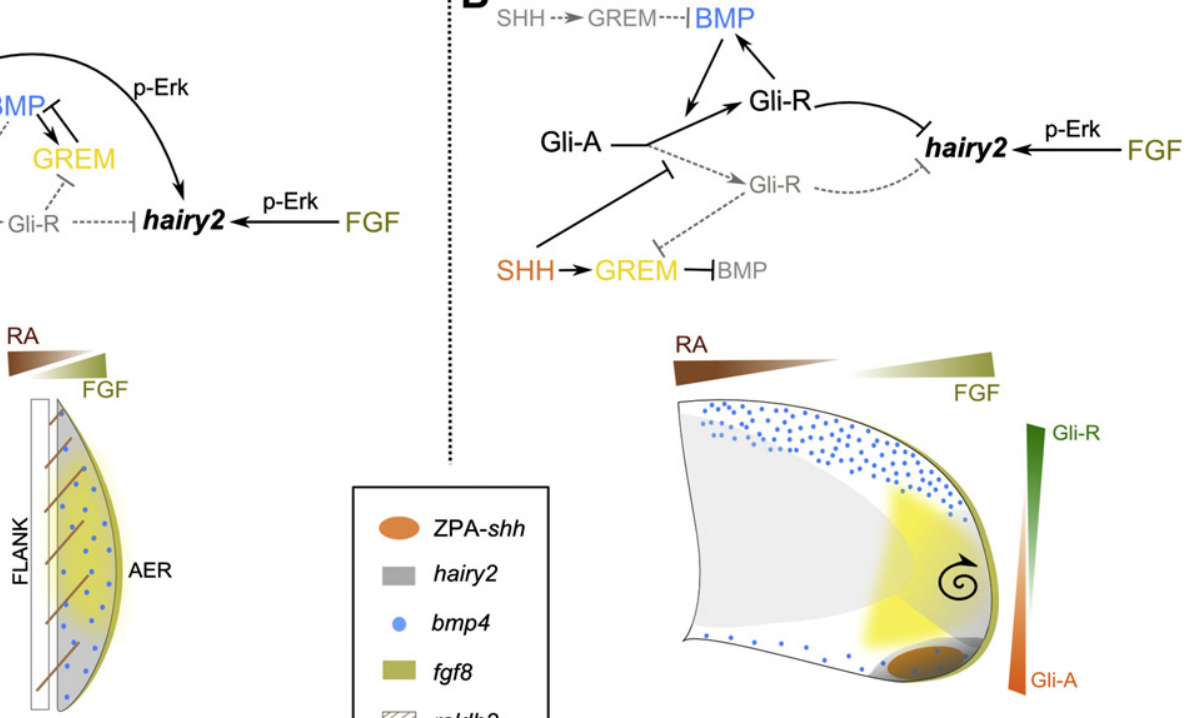

HH17
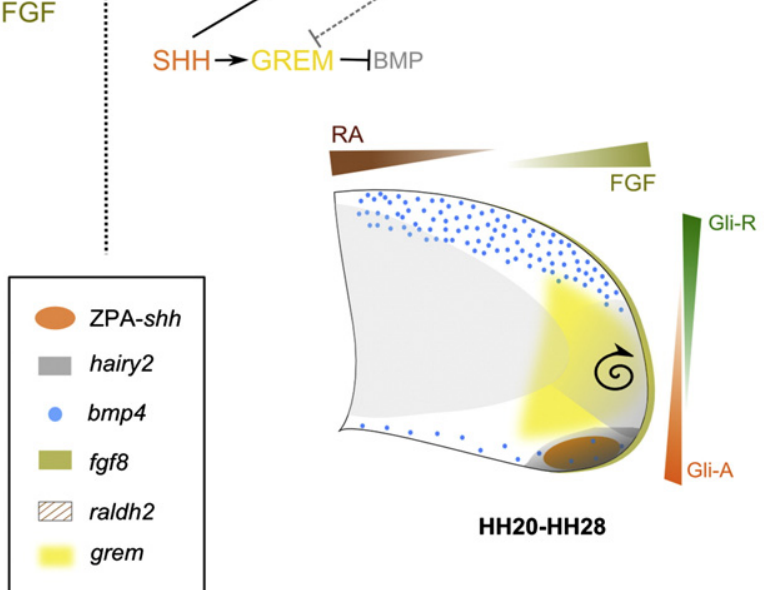

Fig. 1. Integrated time-space model for limb PD/AP patterning: hairy2 expression during limb development reflects the integrated signaling activities mediating both $P D$ and AP limb patterning. $(A)$ In the early limb bud $(H H 17)$, the entire mesenchyme is under the influence of flank-RA and AER-FGF signaling. RA signaling inhibits Gli-R directly and by counteracting BMP activity, establishing a widespread permissive condition (Gli3-A/Gli3-R $>1$ ) for hairy2 induction by both FGF and RA signaling though p-Erk. BMP signaling is further antagonized by GREM. (B) At later stages of limb development $(\mathrm{HH} 20-\mathrm{HH} 28)$, the distal mesenchyme is distanced from the influence of proximal RA. The distal AP axis displays a gradient of Gli-A/Gli-R levels due to polarized SHH signaling from the ZPA. The anterior limb presents high levels of BMP and Gli-R, inhibiting hairy2 expression. Intermediate levels of Gli-A/Gli-R in the medial distal mesenchyme allow for oscillatory hairy2 expression. The presence of GREM in that tissue may contribute to appropriate Gli-A/Gli-R levels by counteracting BMP signaling. In the posterior limb, high levels of Gli-A/Gli-R and FGF produce permanent hairy2 induction. This signaling scenario enables off/oscillatory/on hairy2 patterns, which may underlie cell fate specification over time. hairy2 oscillations in the distal mesenchyme take place in the chondrogenic precursor cells [19] along both PD and AP axes. Since the limb clock is simultaneously integrating molecular information along these axes, it could play a crucial role for coordinated PD/AP limb outgrowth and patterning over time. Anterior is up; posterior is down; proximal is left; distal is right. 
relieving inhibition of BMP activity, ensuring low levels of Gli3-A/Gli3-R. shh expression in the ZPA limits Gli-R levels in the posterior distal limb (Gli-A/ Gli-R > 1) and establishes a gradient of Gli signaling along the limb AP axis [6], which dictates tissue responsiveness to instructive AER-FGF signaling on hairy2. The Gli-A/Gli-R $>1$ permissive condition is further corroborated in the medial distal limb by $\mathrm{SHH}$-induced GREM, which represses BMP activity [34]. Hence, the joint action of permissive ZPA-SHH and instructive AER-FGF now shape hairy2 expression pattern and create the conditions for transcriptional cycles of hairy2 in the medial distal limb domain.

The newly identified regulatory actions of FGF/RA/ $\mathrm{SHH}$ limb morphogens on hairy2 expression suggest that it is possible to conciliate the two-signal and progress zone models, linking spatial morphogenic patterning with temporal precision. Flank-RA signaling, concomitant with non-oscillatory hairy2 expression until stage $\mathrm{HH} 19$ may specify the proximal-most stylopod early in development $[41,42]$. Over time, the distal mesenchyme (progress zone) escapes RA influence and is under continuous AER-FGF and ZPA-SHH signaling gradients. Under these conditions, hairy2 presents oscillatory expression in chondrogenic precursor cells [19], which could constitute a time-counting mechanism progressively providing cell positional information for zeugopod (until stage $\mathrm{HH} 23$ ) and autopod specification [41,42]. Towers et al. have suggested that a switch from a gradientbased to a clock-based molecular mechanism could underlie limb PD patterning [43]. The reports herein revised $[7,8]$ are in agreement with this hypothesis and present hairy2 transcription factor as a crucial molecular component integrating morphogen gradient information along limb PD and AP axes. Moreover, the limb AP off/oscillatory/on states of hairy2 expression in response to graded $\mathrm{SHH}$ morphogen signaling strongly support the model for morphogen interpretation proposed by Balaskas et al. [28] and place hairy2 in the transcriptional network underlying graded Gli activity interpretation in the developing limb.

\section{Acknowledgements}

C.J.S. was supported by Fundação para a Ciência e a Tecnologia, Portugal (grant SFRH/BPD/89493/ 2012); R.P.A. is funded by Ciencia 2007 Program Contract (Portuguese Government) and Programa Operacional Regional do Norte (ON.2) NORTE-070124-FEDER-000017. This work was supported by research grants from Institute for Biotechnology and Bioengineering/Centro de Biomedicina Molecular e Estrutural, LA (to I.P.), by the national Portuguese funding through Fundação para a Ciência e a Tecnologia (National and FEDER COMPETE Program funds: PTDC/SAU-OBD/099758/2008 and
PTDC/SAU-BID/121459/2010 to I.P. and R.P.A., respectively) and by PEst-OE/EQB/LA0023/2011.

Received 19 July 2013; Received in revised form 10 October 2013; Accepted 6 November 2013 Available online 4 December 2013

Keywords: fibroblast growth factors; sonic hedgehog; retinoic acid; molecular clock; limb pattern formation

This is an open-access article distributed under the terms of the Creative Commons AttributionNonCommercial-No Derivative Works License, which permits non-commercial use, distribution, and reproduction in any medium, provided the original author and source are credited.

$\dagger$ R.P.A. and I.P. contributed equally to this work.

Abbreviations used:

$\mathrm{RA}$, retinoic acid; FGF, fibroblast growth factor; $\mathrm{SHH}$, sonic hedgehog; AER, apical ectodermal ridge; PD, proximal-distal; ZPA, zone of polarizing activity; AP, anterior-posterior.

\section{References}

[1] Summerbell D, Lewis JH, Wolpert L. Positional information in chick limb morphogenesis. Nature 1973;244:492-6.

[2] Corson LB, Yamanaka Y, Lai KM, Rossant J. Spatial and temporal patterns of ERK signaling during mouse embryogenesis. Development 2003;130:4527-37.

[3] Kawakami Y, Rodriguez-Leon J, Koth CM, Buscher D, Itoh T, Raya A, et al. MKP3 mediates the cellular response to FGF8 signalling in the vertebrate limb. Nat Cell Biol 2003;5:513-9.

[4] Mercader N, Leonardo E, Piedra ME, Martinez AC, Ros MA, Torres M. Opposing RA and FGF signals control proximodistal vertebrate limb development through regulation of Meis genes. Development 2000;127:3961-70.

[5] Ahn S, Joyner AL. Dynamic changes in the response of cells to positive hedgehog signaling during mouse limb patterning. Cell 2004;118:505-16.

[6] Tickle C, Barker $\mathrm{H}$. The Sonic hedgehog gradient in the developing limb. WIREs Dev Biol 2013;2:275-90.

[7] Sheeba CJ, Andrade RP, Palmeirim I. Joint interpretation of AER/FGF and ZPA/SHH over time and space underlies hairy2 expression in the chick limb. Biol Open 2012;1:1102-10.

[8] Sheeba CJ, Palmeirim I, Andrade RP. Retinoic acid signaling regulates embryonic clock hairy2 gene expression in the developing chick limb. Biochem Biophys Res Commun 2012;423:889-94.

[9] Tabin C, Wolpert L. Rethinking the proximodistal axis of the vertebrate limb in the molecular era. Genes Dev 2007;21:1433-42. 
[10] Mariani FV, Ahn CP, Martin GR. Genetic evidence that FGFs have an instructive role in limb proximal-distal patterning. Nature 2008;453:401-5.

[11] Sun X, Mariani FV, Martin GR. Functions of FGF signalling from the apical ectodermal ridge in limb development. Nature 2002;418:501-8.

[12] Mic FA, Sirbu IO, Duester G. Retinoic acid synthesis controlled by Raldh2 is required early for limb bud initiation and then later as a proximodistal signal during apical ectodermal ridge formation. J Biol Chem 2004;279:26698-706.

[13] Zhao X, Sirbu IO, Mic FA, Molotkova N, Molotkov A, Kumar $S$, et al. Retinoic acid promotes limb induction through effects on body axis extension but is unnecessary for limb patterning. Curr Biol 2009;19:1050-7.

[14] Cooper KL, Hu JK, ten Berge D, Fernandez-Teran M, Ros MA, Tabin CJ. Initiation of proximal-distal patterning in the vertebrate limb by signals and growth. Science 2011;332:1083-6.

[15] Rosello-Diez A, Ros MA, Torres M. Diffusible signals, not autonomous mechanisms, determine the main proximodistal limb subdivision. Science 2011;332:1086-8.

[16] Harfe BD, Scherz PJ, Nissim S, Tian H, McMahon AP, Tabin CJ. Evidence for an expansion-based temporal Shh gradient in specifying vertebrate digit identities. Cell 2004;118:517-28.

[17] Scherz PJ, McGlinn E, Nissim S, Tabin CJ. Extended exposure to Sonic hedgehog is required for patterning the posterior digits of the vertebrate limb. Dev Biol 2007;308:343-54.

[18] Zhu J, Nakamura E, Nguyen MT, Bao X, Akiyama H, Mackem S. Uncoupling Sonic hedgehog control of pattern and expansion of the developing limb bud. Dev Cell 2008;14:624-32.

[19] Pascoal S, Carvalho CR, Rodriguez-Leon J, Delfini MC, Duprez D, Thorsteinsdottir S, et al. A molecular clock operates during chick autopod proximal-distal outgrowth. J Mol Biol 2007;368:303-9.

[20] Pascoal S, Palmeirim I. Watch-ing out for chick limb development. Integr Comp Biol 2007;47:382-9.

[21] Palmeirim I, Henrique D, Ish-Horowicz D, Pourquie O. Avian hairy gene expression identifies a molecular clock linked to vertebrate segmentation and somitogenesis. Cell 1997:91:639-48.

[22] Kageyama R, Niwa Y, Shimojo H, Kobayashi T, Ohtsuka T. Ultradian oscillations in Notch signaling regulate dynamic biological events. Curr Top Dev Biol 2010;92:311-31.

[23] Aulehla A, Pourquie O. Signaling gradients during paraxial mesoderm development. Cold Spring Harbor Perspect Biol 2010;2:a000869.

[24] Saga Y. The synchrony and cyclicity of developmental events. Cold Spring Harbor Perspect Biol 2012;4:a008201.

[25] Resende TP, Ferreira M, Teillet MA, Tavares AT, Andrade RP, Palmeirim I. Sonic hedgehog in temporal control of somite formation. Proc Natl Acad Sci USA 2010;107:12907-12.

[26] Wang C, Ruther U, Wang B. The Shh-independent activator function of the full-length Gli3 protein and its role in vertebrate limb digit patterning. Dev Biol 2007;305:460-9.

[27] McGlinn E, van Bueren KL, Fiorenza S, Mo R, Poh AM, Forrest $A$, et al. Pax9 and Jagged1 act downstream of Gli3 in vertebrate limb development. Mech Dev 2005;122:1218-33.
[28] Balaskas N, Ribeiro A, Panovska J, Dessaud E, Sasai N, Page KM, et al. Gene regulatory logic for reading the Sonic Hedgehog signaling gradient in the vertebrate neural tube. Cell 2012;148:273-84.

[29] Panovska-Griffiths J, Page KM, Briscoe J. A gene regulatory motif that generates oscillatory or multiway switch outputs. J R Soc Interface 2013;10:20120826.

[30] Niwa Y, Masamizu Y, Liu T, Nakayama R, Deng CX, Kageyama R. The initiation and propagation of Hes7 oscillation are cooperatively regulated by Fgf and notch signaling in the somite segmentation clock. Dev Cell 2007;13:298-304.

[31] Nakayama K, Satoh T, Igari A, Kageyama R, Nishida E. FGF induces oscillations of Hes1 expression and Ras/ERK activation. Curr Biol 2008;18:R332-4.

[32] Lee JS, Park JH, Kwon IK, Lim JY. Retinoic acid inhibits BMP4-induced C3H10T1/2 stem cell commitment to adipocyte via downregulating Smad/p38MAPK signaling. Biochem Biophys Res Commun 2011;409:550-5.

[33] Thompson DL, Gerlach-Bank LM, Barald KF, Koenig RJ. Retinoic acid repression of bone morphogenetic protein 4 in inner ear development. Mol Cell Biol 2003;23:2277-86.

[34] Benazet JD, Bischofberger M, Tiecke E, Goncalves A, Martin JF, Zuniga A, et al. A self-regulatory system of interlinked signaling feedback loops controls mouse limb patterning. Science 2009;323:1050-3.

[35] Probst S, Kraemer C, Demougin P, Sheth R, Martin GR, Shiratori $\mathrm{H}$, et al. SHH propagates distal limb bud development by enhancing CYP26B1-mediated retinoic acid clearance via AER-FGF signalling. Development 2011;138:1913-23.

[36] Meyer NP, Roelink $\mathrm{H}$. The amino-terminal region of Gli3 antagonizes the Shh response and acts in dorsoventral fate specification in the developing spinal cord. Dev Biol 2003;257:343-55.

[37] Bastida MF, Delgado MD, Wang B, Fallon JF, Fernandez-Teran M, Ros MA. Levels of Gli3 repressor correlate with Bmp4 expression and apoptosis during limb development. Dev Dyn 2004;231:148-60.

[38] Aoto K, Nishimura T, Eto K, Motoyama J. Mouse GLI3 regulates Fgf8 expression and apoptosis in the developing neural tube, face, and limb bud. Dev Biol 2002;251:320-32.

[39] Lopez-Rios J, Speziale D, Robay D, Scotti M, Osterwalder M, Nusspaumer G, et al. GLI3 constrains digit number by controlling both progenitor proliferation and BMP-dependent exit to chondrogenesis. Dev Cell 2012;22:837-48.

[40] te Welscher P, Fernandez-Teran M, Ros MA, Zeller R. Mutual genetic antagonism involving GLI3 and dHAND prepatterns the vertebrate limb bud mesenchyme prior to $\mathrm{SHH}$ signaling. Genes Dev 2002;16:421-6.

[41] Dudley AT, Ros MA, Tabin CJ. A re-examination of proximodistal patterning during vertebrate limb development. Nature 2002;418:539-44.

[42] Sato K, Koizumi Y, Takahashi M, Kuroiwa A, Tamura K. Specification of cell fate along the proximal-distal axis in the developing chick limb bud. Development 2007;134:1397-406.

[43] Towers M, Wolpert L, Tickle C. Gradients of signalling in the developing limb. Curr Opin Cell Biol 2012;24:181-7. 